\title{
Eficácia do Ondansetron, Metoclopramida, Droperidol e Dexametasona na Prevenção de Náusea e Vômito após Laparoscopia Ginecológica em Regime Ambulatorial. Estudo Comparativo *
}

\section{Efficacy of Ondansetron, Metoclopramide, Droperidol and Dexametasone in Preventing Post-Gynecological Videolaparoscopy Nausea and Vomiting in Outpatient Setting. Comparative Study}

Múcio Paranhos de Abreu, TSA ${ }^{1}$; João Lopes Vieira, TSA ${ }^{2}$; lara Ferreira da Silva ${ }^{3}$; Luiz Eduardo Paula G Miziara ${ }^{3}$; Renata Fófano ${ }^{4}$

\section{RESUMO}

Abreu MP, Vieira JL, Silva IF, Miziara LEPG, Fofano R - Eficácia do Ondansetron, Metoclopramida, Droperidol e Dexametasona na Prevenção de Náusea e Vômito após Laparoscopia Ginecológica em Regime Ambulatorial. Estudo Comparativo

JUSTIFICATIVA E OBJETIVOS: Embora o ondansetron seja apontado como uma das drogas mais eficientes no controle das náuseas e vômitos pós-operatório (NVPO), seu alto custo o torna inviável para uso rotineiro. Este estudo teve como finalidade verificar entre o droperidol, a metoclopramida e a dexametasona qual se aproxima mais da eficácia do ondansetron na prevenção de NVPO em laparoscopias ginecológicas.

MÉTODO: Participaram do estudo 100 pacientes submetidas à laparoscopia ginecológica sob anestesia geral venosa e inalatória, divididas aleatoriamente em cinco grupos de acordo com a medicação antiemética recebida. O grupo GO $(n=20)$ recebeu ondansetron (4 $\mathrm{mg})$; o grupo $G M(n=20)$ : metoclopramida (10 mg); grupo $G D(n=20)$ : droperidol $(1,25$ $m g)$, o grupo $G X(n=20)$ : dexametasona $(8 \mathrm{mg})$ e o grupo $G C$ grupo controle $(n=20)$ não recebeu medicação antiemética. Foram verificadas as incidências de náusea e/ou vômito no pós-operatório, os parâmetros hemodinâmicos, o tempo na sala de recuperação pós-anestésica (SRPA) e o tempo da anestesia.

RESULTADOS: Não houve diferença estatística entre os grupos quanto aos dados antropométricos, hemodinâmicos, tempo de recuperação e tempo de anestesia. Houve diferença estatística entre os grupos quanto à incidência de náusea (GO

\footnotetext{
* Recebido do (Received from) Hospital Centro Médico de Campinas e Instituto Penido Burnier, Campinas, SP

1. Instrutor do CET/SBA do Instituto Penido Burnier e Centro Médico de Campinas

2. Professor Doutor Coordenador da Disciplina de Anestesiologia do CCMB da PUC-SP, Co-Responsável pelo CET/SBA do Instituto Penido Burnier e Centro Médico de Campinas.

3. $M E_{2}$ (2003) do CET/SBA

4. $M E_{2}$ (2002) do CET/SBA
}

Apresentado (Submitted) em 27 de junho de 2005

Aceito (Accepted) para publicação em 22 de novembro de 2005

Endereço para correspondência (Correspondence to)

Dr. Múcio Paranhos de Abreu

Av. Nossa Senhora de Fátima, 805/J-131 Taquaral

13090-000 Campinas, SP

E-mail:mpabreu2@gmail.com

(C) Sociedade Brasileira de Anestesiologia, 2006
$<G D<G X<G M<G C)$ e de vômitos $(G O<G D<G X<G M<$ $G C)$.

CONCLUSÕES: Neste estudo, o ondansetron foi o agente mais eficaz na profilaxia de náusea e vômito e o droperidol foi a droga que mais se aproximou da eficácia do ondansetron na prevenção de NVPO.

Unitermos: ANTIEMÉTICOS: dexametasona, droperidol, metoclopramida, ondansetron; CIRURGIA, Ginecológica: laparoscopia; COMPLICAÇÕES: náusea, vômito

\section{SUMMARY}

Abreu MP, Vieira JL, Silva IF, Miziara LEPG, Fofano R - Efficacy of Ondansetron, Metoclopramide, Droperidol and Dexametasone in Preventing Post-Gynecological Videolaparoscopy Nausea and Vomiting in Outpatient Setting. Comparative Study

BACKGROUND AND OBJECTIVES: Although being considered one of the most effective drugs to control postoperative nausea and vomiting (PONV), ondansetron is unfeasible for routine use due to its high cost. This study aimed at comparing the efficacy of droperidol, metoclopramide, and dexametasone as compared to ondansetron in preventing PONV after gynecological laparoscopies.

METHODS: Participated in the study 100 patients submitted to gynecological laparoscopies under general intravenous and inhalational anesthesia, who were randomly distributed in five groups according to the antiemetic medication. Group GO ( $n=$ 20) received ondansetron (4 mg); Group $G M(n=20)$ received metoclopramide $(10 \mathrm{mg})$; Group $G D(n=20)$ received droperidol $(1.25 \mathrm{mg})$; Group $G X(n=20)$ received dexametasone (8 mg); and Group GC - control group $(n=20)$ was not medicated. The following events were recorded: incidence of postoperative nausea and/or vomiting, hemodynamic parameters, PACU stay and anesthetic length.

RESULTS: There were no statistically significant differences among groups in demographics, hemodynamic parameters, recovery and anesthetic length. There were statistical differences among groups in the incidence of nausea ( $G O<G D<G X<G M$ $<G C)$ and vomiting $(G O<G D<G X<G M<G C)$.

CONCLUSIONS: Ondansetron was the most effective agent in preventing nausea and vomiting, and droperidol was the closest drug to ondansetron in preventing PONV.

Key Words: ANTIEMETICS: dexametasone, droperidol, metoclopramide, ondansetron; COMPLICATIONS: nausea, vomiting; SURGERY: Gynecological: laparoscopic 


\section{INTRODUÇÃO}

$\mathrm{N}$ áuseas e vômitos pós-operatórios (NVPO) são os efeitos adversos mais comuns que podem aparecer após procedimentos anestésico-cirúrgicos, especialmente nas laparoscopias ginecológicas ${ }^{1-5}$. Embora a etiologia da NVPO não esteja completamente definida, sabe-se que ela tem caráter multifatorial ${ }^{4}$. Os fatores que podem aumentar a ocorrência de NVPO incluem aqueles relacionados ao paciente como: sexo; idade; peso; ansiedade e história prévia de NVPO, entre outros; e relacionados à cirurgia e à técnica anestésica como: o local e a duração da cirurgia, ventilação sob máscara e utilização de drogas com potencial emetogênico. Pacientes jovens submetidas à laparoscopia ginecológica diagnóstica ou cirúrgica, constituem um grupo de risco elevado para desenvolverem NVPO ${ }^{6,7}$

Alguns estudos comprovaram a eficácia do ondansetron na profilaxia e tratamento da NVPO ${ }^{8,9}$, no entanto o custo elevado deste fármaco torna inviável o seu uso rotineiro para esse fim. Outros estudos mostraram que baixas doses de droperidol $(0,625$ a $1,25 \mathrm{mg})$ apresentam bom efeito antiemético, embora limitado, nos procedimentos mais emetogênicos ${ }^{10}$. Apesar da literatura não ser conclusiva quanto à dose ideal de droperidol, a dose de $1,25 \mathrm{mg}$ tem se mostrado satisfatório.

A metoclopramida, muito utilizada na prevenção de estímulo de receptores dopaminérgicos e na aceleração do esvaziamento gástrico, não tem se mostrado muito eficaz como antiemético em cirurgia laparoscópica.

A dexametasona e outros glicocorticóides parecem possuir efeitos antieméticos, melhorando a eficácia da terapia antiemética.

As cirurgias ginecológicas por videolaparoscopia têm apresentado alta incidência de vômitos no pós-operatório e nas pacientes com fatores de risco associados para apresentarem NVPO a profilaxia se faz necessária.

O objetivo deste trabalho foi verificar qual das drogas utilizadas neste estudo: droperidol, dexametasona e metoclopramida, mais se aproximam da eficácia do ondansetron na profilaxia e tratamento da NVPO em laparoscopia ginecológica.

\section{MÉTODO}

Após aprovação pela Comissão de Ética do Centro Médico de Campinas e consentimento formal, participaram do estudo, 100 pacientes com idade entre 22 e 62 anos, estado físico ASAI e II, submetidas à laparoscopia ginecológica diagnóstica ou cirúrgica sob anestesia geral venosa e inalatória. Foram excluídas as pacientes com história prévia de náusea e vômito pós-operatório, bem como aquelas portadoras de distúrbios gástricos. As pacientes foram divididas aleatoriamente em cinco grupos de acordo com a droga antiemética, administrada imediatamente após a indução anestésica: grupo GO: ondansetron (4 mg); grupo GM: metoclopramida (10 mg); grupo GD: droperidol (1,25 mg); grupo GX: dexametasona (8 $\mathrm{mg}$ ) e grupo GC (controle) que não recebeu medicação antiemética.
Após jejum de no mínimo oito horas, as pacientes foram encaminhadas à sala de cirurgia, realizada venóclise com cateter $20 \mathrm{G}$ no membro superior esquerdo, seguida da administração de solução de Ringer com lactato $\left(10 \mathrm{~mL} \cdot \mathrm{kg}^{-1} \cdot \mathrm{h}^{-1}\right)$. A monitorização foi realizada com cardioscópio na derivação $D_{\text {II }}$, aferição automática não-invasiva de pressão arterial, oxímetro de pulso, capnógrafo e análise de gases expirados. A indução anestésica foi realizada com midazolam $(0,05$ $\left.\mathrm{mg} \cdot \mathrm{kg}^{-1}\right)$, fentanil $\left(3 \mu \mathrm{g} \cdot \mathrm{kg}^{-1}\right)$, lidocaína a $2 \%\left(1 \mathrm{mg} \cdot \mathrm{kg}^{-1}\right)$, propofol $\left(1,5 \mathrm{mg} \cdot \mathrm{kg}^{-1}\right)$ e atracúrio $\left(0,5 \mathrm{mg} \cdot \mathrm{kg}^{-1}\right)$ para todas as pacientes. Após ventilação manual com oxigênio a $100 \%$, procedeu-se a intubação traqueal e a manutenção da anestesia foi realizada com $\mathrm{N}_{2} \mathrm{O}: \mathrm{O}_{2}$ 1:1 e isoflurano (1 CAM), em sistema de ventilação mecânica com reabsorção de dióxido de carbono. Afreqüência respiratória foi regulada para mantera pressão expirada de dióxido de carbono $\left(\mathrm{P}_{\mathrm{ET}} \mathrm{CO}_{2}\right)$ em torno de $37 \mathrm{mmHg}$. Foram administrados $50 \mu \mathrm{g}$ de fentanil sempre que necessário, para manutenção do plano anestésico. A analgesia pós-operatória foi realizada com cetoprofeno (100 $\mathrm{mg}$ ). Ao final da cirurgia e após recuperação da ventilação espontânea efetiva, as pacientes foram extubadas e encaminhadas à sala de recuperação pós-anestésica (SRPA). Não houve necessidade de realizar reversão do bloqueio neuromuscular em nenhuma paciente. A pressão arterial sistólica (PAS), diastólica (PAD) e freqüência cardíaca (FC) foram anotadas em protocolo para tratamento estatístico, nos seguintes momentos:

M1 = Antes da indução;

M2 = Após intubação orotraqueal;

M3 = Antes da insuflação peritoneal;

M4 = 10 minutos após insuflação;

M5 = 20 minutos após insuflação;

M6 = Final da anestesia;

M7 = Alta da SRPA.

Durante a permanência na SRPA, um anestesiologista avaliava a presença de náusea e/ou vômito periodicamente, registrando os resultados. Após receberem alta da SRPA, as pacientes foram encaminhadas para a unidade ambulatorial, onde permaneceram por aproximadamente quatro horas, até a alta hospitalar. Foi verificado se alguma paciente permaneceu mais tempo na Unidade Ambulatorial devido à ocorrência de náuseas e vômito. Foram verificados também o tempo de anestesia, os dados hemodinâmicos e o tempo de permanência na SRPA. Durante a permanência na unidade ambulatorial as pacientes foram inquiridas quanto à presença de náuseas e/ou vômitos e anotados os resultados. Foi utilizado o teste $t$ Student para comparação das variáveis: peso, altura e idade e a prova não paramétrica de Kruskal-Wallis, para amostras independentes para comparar a incidência de náusea e vômito, estabelecido o valor de $p<0,05$ como significativo.

Os dados hemodinâmicos (pressão arterial e freqüência cardíaca) foram codificados, digitalizados e analisados com o software de estatística distribuído pela Organização Mundial de Saúde (OMS), Epi-Info versão 6.04. 


\section{RESULTADOS}

Os grupos foram homogêneos quanto aos dados antropométricos e estado físico (ASA) (Tabela I).
Não houve diferenças estatísticas significativas entre os grupos quanto aos dados hemodinâmicos (Tabelas II e III) e quantidade de drogas utilizadas por via venosa (Tabela IV). Não houve diferença estatística entre os grupos quanto ao tempo de anestesia e de permanência na SRPA(Tabela V).

Tabela I - Dados Antropométricos dos Grupos (Média \pm DP)

\begin{tabular}{lccccc}
\hline Variáveis & Grupo GO & Grupo GD & Grupo GM & Grupo GX & Grupo GC \\
\hline Idade (anos) & $34,53 \pm 5,50$ & $34,23 \pm 9,24$ & $34,30 \pm 7,78$ & $31,56 \pm 5,58$ & $35,60 \pm 6,26$ \\
Peso (kg) & $60,58 \pm 10,12$ & $62,82 \pm 10,04$ & $64,43 \pm 13,15$ & $59,43 \pm 12,75$ & $57,52 \pm 9,58$ \\
Altura (cm) & $161,89 \pm 5,87$ & $163,90 \pm 7,40$ & $162,35 \pm 6,23$ & $162,95 \pm 5,05$ & $162,26 \pm 6,94$ \\
Estado físico & 14 & & & & 17 \\
\multicolumn{1}{c}{ ASA I } & 6 & 16 & 16 & 3 & 4 \\
ASAll & 4 & 4 & 4 & 3 & 4 \\
\hline
\end{tabular}

Não houve diferença estatística entre os grupos

Tabela II - Pressão Arterial Sistólica dos Grupos (Média \pm DP)

\begin{tabular}{lccccc}
\hline Momentos & Grupo GO & Grupo GD & Grupo GM & Grupo GX & Grupo GC \\
\hline M1 & $112,7 \pm 17,7$ & $113,3 \pm 13,7$ & $105,6 \pm 11,4$ & $127,8 \pm 58,1$ & $105,8 \pm 8,7$ \\
M2 & $124 \pm 16,9$ & $114,7 \pm 24,8$ & $113,9 \pm 13$ & $110,8 \pm 16,8$ & $112,5 \pm 12,3$ \\
M3 & $106,6 \pm 13,2$ & $111,3 \pm 19,6$ & $99,4 \pm 16,3$ & $107,3 \pm 14,6$ & $103,6 \pm 12,7$ \\
M4 & $98,70 \pm 12,5$ & $96,5 \pm 14,8$ & $89,7 \pm 11,3$ & $97,8 \pm 10,5$ & $99,4 \pm 11,9$ \\
M5 & $100,2 \pm 27,7$ & $107,7 \pm 10,5$ & $99,7 \pm 12,4$ & $111,2 \pm 12,7$ & $105,3 \pm 10,7$ \\
M6 & $122,5 \pm 16$ & $116,6 \pm 10,4$ & $121,6 \pm 10,5$ & $120,6 \pm 10,8$ & $121,8 \pm 10,3$ \\
M7 & $118,3 \pm 12,6$ & $116,7 \pm 9,8$ & $115,7 \pm 12$ & $117,2 \pm 11,3$ & $114,9 \pm 11,8$ \\
\hline
\end{tabular}

Não houve diferença estatística entre os grupos

Tabela III - Pressão Arterial Diastólica dos Grupos (Média \pm DP)

\begin{tabular}{lccccc}
\hline Momentos & Grupo GO & Grupo GD & Grupo GM & Grupo GX & Grupo GC \\
\hline M1 & $71,3 \pm 8,5$ & $75,6 \pm 11$ & $68,3 \pm 8,6$ & $72,87 \pm 11,1$ & $74,4 \pm 9,9$ \\
M2 & $76,7 \pm 9,2$ & $73,3 \pm 10,9$ & $72,4 \pm 9,7$ & $67,2 \pm 10$ & $62,8 \pm 11,7$ \\
M3 & $68,2 \pm 11,9$ & $69 \pm 14,8$ & $62,5 \pm 13,4$ & $70,6 \pm 11,5$ & $72,6 \pm 9,3$ \\
M4 & $62 \pm 9$ & $61,2 \pm 12,1$ & $54,5 \pm 8$ & $63,6 \pm 10,1$ & $62,8 \pm 11,3$ \\
M5 & $70,6 \pm 11,9$ & $67,3 \pm 9,8$ & $70,31 \pm 13,6$ & $68,9 \pm 11,1$ & $69,6 \pm 11,5$ \\
M6 & $78,5 \pm 11,9$ & $79,1 \pm 8,9$ & $80 \pm 12,6$ & $79,7 \pm 11,7$ & $77,9 \pm 11,2$ \\
M7 & $75,3 \pm 8$ & $77,4 \pm 9,7$ & $73,16 \pm 12,4$ & $74,7 \pm 8,7$ & $76,8 \pm 8,6$ \\
\hline
\end{tabular}

Não houve diferença estatística entre os grupos

Tabela IV - Drogas Anestésicas Utilizadas (Média \pm DP)

\begin{tabular}{|c|c|c|c|c|c|}
\hline Drogas & Grupo GO & Grupo GD & Grupo GM & Grupo GX & Grupo GC \\
\hline Midazolam (mg) & $2,67 \pm 0,9$ & $2,89 \pm 1$ & $2,89 \pm 0,8$ & $3,34 \pm 1,1$ & $2,93 \pm 1$ \\
\hline Lidocaína (mg) & $72,38 \pm 10,5$ & $97,36 \pm 44,6$ & $94,73 \pm 33$ & $72,63 \pm 22$ & $86,31 \pm 58,5$ \\
\hline Fentanil (mg) & $175 \pm 44,7$ & $190,52 \pm 40,6$ & $175 \pm 39,9$ & $188,16 \pm 38,5$ & $180,78 \pm 39,2$ \\
\hline Propofol (mg) & $85,71 \pm 13,9$ & $91,05 \pm 17,9$ & $90,52 \pm 15,4$ & $91,58 \pm 18,6$ & $94,73 \pm 33,7$ \\
\hline Atracúrio (mg) & $30 \pm 13,6$ & $28,73 \pm 6,6$ & $30,26 \pm 4,5$ & $28,16 \pm 6,3$ & $27,89 \pm 6,3$ \\
\hline
\end{tabular}

Não houve diferença estatística entre os grupos 
Tabela V - Tempo de Anestesia e Tempo de Permanência na Sala de Recuperação Pós-Anestésica (Média \pm DP)

\begin{tabular}{lccccc}
\hline & Grupo GO & Grupo GD & Grupo GM & Grupo GX & Grupo GC \\
\hline Tempo de anestesia (min) & $103,33 \pm 33,2$ & $93,42 \pm 26,88$ & $80,53 \pm 20,06$ & $89,74 \pm 15,05$ & $100,37 \pm 24,35$ \\
Tempo de SRPA (min) & $161,89 \pm 5,87$ & $163,90 \pm 7,40$ & $162,35 \pm 6,23$ & $162,95 \pm 5,05$ & $162,26 \pm 6,94$ \\
\hline
\end{tabular}

Não houve diferença estatística entre os grupos

Quanto à incidência de náuseas no pós-operatório houve diferença estatística entre os grupos: $\mathrm{GO}<\mathrm{GD}<\mathrm{GX}<\mathrm{GM}<\mathrm{GC}$ (Figura 1).

Quanto à incidência de vômitos no pós-operatório houve diferença estatística entre os grupos: $\mathrm{GO}<\mathrm{GD}<\mathrm{GX}<\mathrm{GM}<\mathrm{GC}$ (Figura 2).

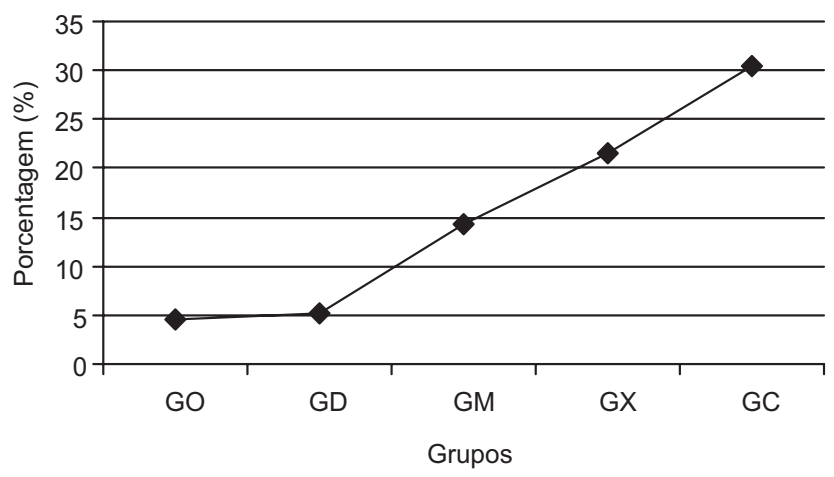

Figura 1 - Incidência de Náuseas

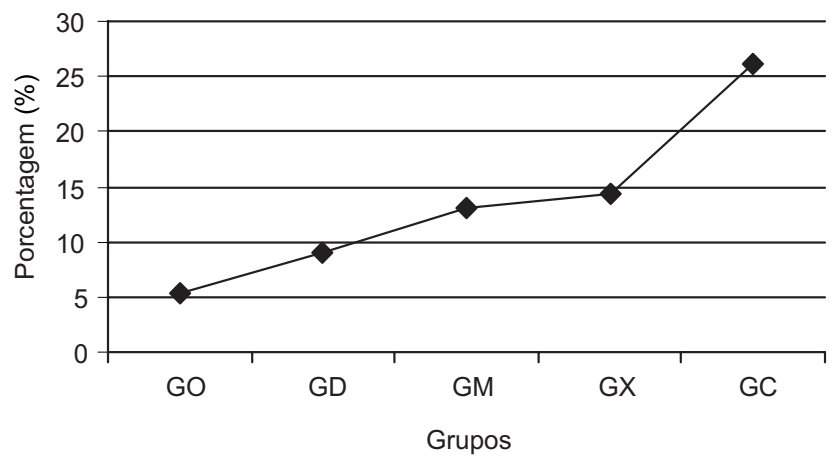

Figura 2 - Incidência de Vômitos

Revista Brasileira de Anestesiologia

Vol. 56, № 1, Janeiro - Fevereiro, 2006

\section{DISCUSSÃO}

A alta incidência de vômitos em cirurgia videolaparoscópica ginecológica ficou evidente também neste estudo, haja vista que as pacientes do grupo controle apresentaram vômito, significando incidência de $26,09 \%$. No entanto, após os episódios de vômito no pós-operatório e o tratamento com metoclopramida, não houve prolongamento da permanência na SRPA e tampouco da permanência hospitalar, ou seja, não houve diferença significativa no tempo de alta da SRPA que corresponde à recuperação do estágio II da anestesia geral. As pacientes foram encaminhadas para a recuperação da unidade ambulatorial (recuperação-2) onde permaneceram até a alta hospitalar (recuperação do estágio III da anestesia geral $)^{11,12}$, quando já não mais apresentavam náuseas e/ou vômitos. A uniformidade dos grupos em relação à alta da SRPA mostrou também que não houve influência dos antieméticos, especialmente o droperidol, na dose utilizada, no tempo de permanência na SRPA. A alta hospitalar dentro do tempo estimado de quatro horas após a alta da SRPA foi possível em todos os grupos, mostrando também a eficiência da técnica anestésica.

O ondansetron se mostrou como a droga mais eficaz na prevenção de náusea (incidência de $4,55 \%$ ) e vômitos $(5,26 \%$ ) no pós-operatório, fato que vem ao encontro dos resultados obtidos por outros autores ${ }^{8-10,13-15}$. O droperidol foi a droga, dentre as estudadas, que mais se aproximou do ondansetron na profilaxia das náuseas $(5,27 \%)$ e vômitos $(9,09 \%)$ em cirurgia videolaparoscópica ginecológica, nas condições deste estudo.

A metoclopramida e a dexametasona foram, nesta ordem, as menos eficazes. Usadas isoladamente não apresentaram resultados satisfatórios, haja vista que nenhuma delas conseguiu diminuir significativamente a incidência de vômitos em relação ao grupo controle. Sabe-se que a etiologia da náusea e do vômito tem caráter multifatorial ${ }^{2,7}$ e está relacionada com quatro tipos de neurotransmissores que modulam a zona quimiorreceptora de gatilho, situada na área postrema: dopamina, serotonina, histamina e acetilcolina ${ }^{9}$. Os antieméticos são classificados de acordo com a ação sobre os receptores farmacológicos e geralmente a monoterapia pode não ser suficiente para controlar as NVPO. Alguns autores sugerem a associação de dois ou mais agentes antieméticos para obter melhores resultados ${ }^{16-18}$. Um estudo mostrou que o uso profilático de granisetron ( $3 \mathrm{mg}$ ) associado a dexametasona ( $8 \mathrm{mg}$ ), administrados por via venosa, foram mais efetivos que o granisetron isoladamente, na redução de NVPO em pacientes submetidas à operação cesaria- 
na sob anestesia espinhal ${ }^{16}$. Os mesmos autores concluíram, em outro estudo, que a terapia profilática com granisetron e dexametasona foi mais efetiva que cada antiemético empregado separadamente, para prevenção de vômitos após cirurgias pediátricas ${ }^{17}$. Outros autores mostraram que a associação de droperidol (1,25 mg) e ondansetron (4 mg), administrados por via venosa, foi significativamente superior ao emprego do droperidol isoladamente, no controle de NVPO em mulheres submetidas à cirurgia ginecológica laparoscópica ${ }^{18}$. Neste estudo não foi feita associação de drogas antieméticas visto que a finalidade era a de estudá-las isoladamente. Assim, nas condições deste estudo, em que os antieméticos foram empregados isoladamente, o ondansetron foi o agente mais eficaz na profilaxia de náusea e vômito e o droperidol foi a droga que mais se aproximou da eficácia daquele, apresentando-se como uma boa opção na prevenção de náuseas e vômitos em cirurgia videolaparoscópica ginecológica em regime ambulatorial.

\section{Efficacy of Ondansetron, Metoclopramide, Droperidol and Dexametasone in Preventing Post-Gynecological Videolapa- roscopy Nausea and Vomiting in Outpatient Setting. Comparative Study}

Múcio Paranhos de Abreu, TSA, M.D.; João Lopes Vieira, TSA, M.D.; Iara Ferreira da Silva, M.D.; Luiz Eduardo Paula G Miziara, M.D.; Renata Fófano, M.D.

\section{INTRODUCTION}

Postoperative nausea and vomiting (PONV) are the most common adverse effects after anesthetic-surgical procedures, especially gynecological laparoscopies ${ }^{1-5}$. Although its etiology is not fully defined, it is known that PONV is a multifactorial event ${ }^{4}$. Factors increasing the incidence of PONV are patient-related, such as gender, age, weight, anxiety and previous PONV history, among others; and procedure-related, such as surgery site and length, ventilation under mask and potentially emetogenic drugs. Young patients submitted to diagnostic or surgical gynecological laparoscopy are at high risk for developing PONV ${ }^{6,7}$.

Some studies have proven the efficacy of ondansetron in preventing and treating PONV ${ }^{8,9}$, however its high cost prevents its routine use. Other studies have shown that low droperidol doses ( 0.625 to $1.25 \mathrm{mg}$ ) have satisfactory, although limited antiemetic effect in highly emetogenic procedures ${ }^{10}$. Literature is inconclusive as to the optimal droperidol dose, but 1.25 $\mathrm{mg}$ has been considered satisfactory.

Metoclopramide, widely used to prevent dopaminergic receptors stimulation and to speed gastric emptying, is not very effective in preventing PONV after laparoscopic procedures.
Dexametasone and other glucocorticoids seem to have antiemetic effects, improving the efficacy of antiemetic therapies.

Videolaparoscopic gynecological procedures have a high incidence of postoperative vomiting and prevention is needed for patients with associated risk factors for PONV.

This study aimed at evaluating which of the following drugs: droperidol, dexametasone and metoclopramide, was closest to ondansetron in preventing and treating PONV after gynecological laparoscopy.

\section{METHODS}

After the institution's Ethic Committee approval and their formal consent, participated in this study 100 patients aged 22 to 62 years, physical status ASA I and II, submitted to diagnostic or surgical gynecological laparoscopy under general intravenous and inhalational anesthesia. Exclusion criteria were patients with previous history of postoperative nausea and vomiting and those with gastric disorders. Patients were randomly distributed in five groups according to the antiemetic drug, administered immediately after anesthetic induction: group GO: ondansetron (4 mg); group GM: metoclopramide (10 mg); group GD: droperidol (1.25 mg); group GX: dexametasone (8 mg) and group GC (control) not medicated. After minimum 8-hour fast, patients were referred to the operating room, intravenous $20 \mathrm{G}$ catheter was inserted in left arm and lactated Ringer's solution was started $\left(10 \mathrm{~mL} \cdot \mathrm{kg}^{-1} \cdot \mathrm{h}^{-1}\right)$. Monitoring consisted of cardioscope at $D_{\|}$lead, noninvasive blood pressure, pulse oximeter, capnography and expired gases analysis. Anesthesia was induced with midazolam $\left(0.05 \mathrm{mg} \cdot \mathrm{kg}^{-1}\right)$, fentanyl $\left(3 \mu \mathrm{g} \cdot \mathrm{kg}^{-1}\right), 2 \%$ lidocaine $\left(1 \mathrm{mg} \cdot \mathrm{kg}^{-1}\right)$, propofol $\left(1.5 \mathrm{mg}^{\mathrm{kg}} \mathrm{kg}^{-1}\right)$ and atracurium $\left(0.5 \mathrm{mg} \cdot \mathrm{kg}^{-1}\right)$ for all patients. After manual ventilation with $100 \%$ oxygen patients were intubated and anesthesia was maintained with $\mathrm{N}_{2} \mathrm{O}: \mathrm{O}_{2}$ 1:1 and isoflurane (1 MAC), under mechanical ventilation with carbon dioxide reabsorption.

Respiratory rate was adjusted to maintain $\mathrm{P}_{\mathrm{ET}} \mathrm{CO}_{2}$ in approximately $37 \mathrm{mmHg}$. Whenever necessary, $50 \mu \mathrm{g}$ fentanyl was administered. Postoperative analgesia was achieved with ketoprofen $(100 \mathrm{mg})$. At surgery completion patients were extubated after effective spontaneous ventilation recovery and were referred to the post-anesthetic recovery unit (PACU). No patient needed neuromuscular reversal. Systolic (SBP) and diastolic (DBP) blood pressure and heart rate $(\mathrm{HR})$ were recorded for statistical analysis in the following moments:

$\mathrm{M} 1$ = Before induction;

M2 = After tracheal intubation;

M3 = Before peritoneal inflation;

M4 = 10 minutes after inflation;

M5 = 20 minutes after inflation;

M6 = End of anesthesia;

M7 = PACU discharge. 
During PACU stay, the presence of nausea and/or vomiting was periodically evaluated and recorded by an anesthesiologist. After PACU discharge, patients were referred to the outpatient setting where they remained for approximately four hours until hospital discharge. Longer outpatient setting stay due to nausea and vomiting was observed. Anesthetic length, hemodynamic parameters and PACU stay were also observed. During outpatient setting stay, patients were asked about the presence of nausea and/or vomiting and results were recorded. Student's $t$ test was used to compare weight, height and age, and non-parametric Kruskal-Wallis test for independent samples was used to compare the incidence of nausea and vomiting, considering significant $p<$ 0.05 .

Hemodynamic parameters (blood pressure and heart rate) were coded, digitized and analyzed with the World Health Organization (WHO) statistical software Epi-Info, release 6.04.

\section{RESULTS}

Groups were homogeneous in demographics data and physical status (ASA) (Table I).

There were no statistically significant differences among groups in hemodynamic parameters (Tables II and III) and intravenous drug doses (Table IV).

There were no statistical differences among groups in anesthetic length and PACU stay (Table V).

There was statistical difference among groups in the incidence of postoperative nausea: $\mathrm{GO}<\mathrm{GD}<\mathrm{GX}<\mathrm{GM}<\mathrm{GC}$ (Figure 1).

There was statistical difference among groups in the incidence of postoperative vomiting: $\mathrm{GO}<\mathrm{GD}<\mathrm{GX}<\mathrm{GM}<\mathrm{GC}$ (Figure 2).

Table I - Demographics Data (Mean \pm SD)

\begin{tabular}{lccccc}
\hline Variables & Group GO & Group GD & Group GM & Group GX & Group GC \\
\hline Age (years) & $34.53 \pm 5.50$ & $34.23 \pm 9.24$ & $34.30 \pm 7.78$ & $31.56 \pm 5.58$ & $35.60 \pm 6.26$ \\
Weight $(\mathrm{kg})$ & $60.58 \pm 10.12$ & $62.82 \pm 10.04$ & $64.43 \pm 13.15$ & $59.43 \pm 12.75$ & $57.52 \pm 9.58$ \\
Height (cm) & $161.89 \pm 5.87$ & $163.90 \pm 7.40$ & $162.35 \pm 6.23$ & $162.95 \pm 5.05$ & $162.26 \pm 6.94$ \\
Physical status & 14 & & & & 17 \\
\multicolumn{1}{c}{ ASA I } & 6 & 16 & 16 & 3 & 16 \\
\multicolumn{1}{c}{ ASAll } & 4 & 4 & 4 & 4 \\
\hline
\end{tabular}

There were no statistical differences among groups

Table II - Systolic Blood Pressure (Mean \pm SD)

\begin{tabular}{|c|c|c|c|c|c|}
\hline Moments & Group GO & Group GD & Group GM & Group GX & Group GC \\
\hline M1 & $112.7 \pm 17.7$ & $113.3 \pm 13.7$ & $105.6 \pm 11.4$ & $127.8 \pm 58.1$ & $105.8 \pm 8.7$ \\
\hline M2 & $124 \pm 16.9$ & $114.7 \pm 24.8$ & $113.9 \pm 13$ & $110.8 \pm 16.8$ & $112.5 \pm 12.3$ \\
\hline M3 & $106.6 \pm 13.2$ & $111.3 \pm 19.6$ & $99.4 \pm 16.3$ & $107.3 \pm 14.6$ & $103.6 \pm 12.7$ \\
\hline M4 & $98.70 \pm 12.5$ & $96.5 \pm 14.8$ & $89.7 \pm 11.3$ & $97.8 \pm 10.5$ & $99.4 \pm 11.9$ \\
\hline M5 & $100.2 \pm 27.7$ & $107.7 \pm 10.5$ & $99.7 \pm 12.4$ & $111.2 \pm 12.7$ & $105.3 \pm 10.7$ \\
\hline M6 & $122.5 \pm 16$ & $116.6 \pm 10.4$ & $121.6 \pm 10.5$ & $120.6 \pm 10.8$ & $121.8 \pm 10.3$ \\
\hline M7 & $118.3 \pm 12.6$ & $116.7 \pm 9.8$ & $115.7 \pm 12$ & $117.2 \pm 11.3$ & $114.9 \pm 11.8$ \\
\hline
\end{tabular}

There were no statistical differences among groups

Table III - Diastolic Blood Pressure (Mean \pm SD)

\begin{tabular}{|c|c|c|c|c|c|}
\hline Moments & Group GO & Group GD & Group GM & Group GX & Group GC \\
\hline M1 & $71.3 \pm 8.5$ & $75.6 \pm 11$ & $68.3 \pm 8.6$ & $72.87 \pm 11.1$ & $74.4 \pm 9.9$ \\
\hline M2 & $76.7 \pm 9.2$ & $73.3 \pm 10.9$ & $72.4 \pm 9.7$ & $67.2 \pm 10$ & $62.8 \pm 11.7$ \\
\hline M3 & $68.2 \pm 11.9$ & $69 \pm 14.8$ & $62.5 \pm 13.4$ & $70.6 \pm 11.5$ & $72.6 \pm 9.3$ \\
\hline M4 & $62 \pm 9$ & $61.2 \pm 12.1$ & $54.5 \pm 8$ & $63.6 \pm 10.1$ & $62.8 \pm 11.3$ \\
\hline M5 & $70.6 \pm 11.9$ & $67.3 \pm 9.8$ & $70.31 \pm 13.6$ & $68.9 \pm 11.1$ & $69.6 \pm 11.5$ \\
\hline M6 & $78.5 \pm 11.9$ & $79.1 \pm 8.9$ & $80 \pm 12.6$ & $79.7 \pm 11.7$ & $77.9 \pm 11.2$ \\
\hline M7 & $75.3 \pm 8$ & $77.4 \pm 9.7$ & $73.16 \pm 12.4$ & $74.7 \pm 8.7$ & $76.8 \pm 8.6$ \\
\hline
\end{tabular}

There were no statistical differences among groups 
ABREU, VIEIRA, SILVA ET AL

Table IV - Anesthetic Drugs Used (Mean \pm SD)

\begin{tabular}{|c|c|c|c|c|c|}
\hline Drugs & Group GO & Group GD & Group GM & Group GX & Group GC \\
\hline Midazolam (mg) & $2.67 \pm 0.9$ & $2.89 \pm 1$ & $2.89 \pm 0.8$ & $3.34 \pm 1.1$ & $2.93 \pm 1$ \\
\hline Lidocaine (mg) & $72.38 \pm 10.5$ & $97.36 \pm 44.6$ & $94.73 \pm 33$ & $72.63 \pm 22$ & $86.31 \pm 58.5$ \\
\hline Fentanyl (mg) & $175 \pm 44.7$ & $190.52 \pm 40.6$ & $175 \pm 39.9$ & $188.16 \pm 38.5$ & $180.78 \pm 39.2$ \\
\hline Propofol (mg) & $85.71 \pm 13.9$ & $91.05 \pm 17.9$ & $90.52 \pm 15.4$ & $91.58 \pm 18.6$ & $94.73 \pm 33.7$ \\
\hline Atracurium (mg) & $30 \pm 13.6$ & $28.73 \pm 6.6$ & $30.26 \pm 4.5$ & $28.16 \pm 6.3$ & $27.89 \pm 6.3$ \\
\hline
\end{tabular}

There were no statistical differences among groups

Table V - Anesthesia Length and PACU stay (Mean \pm SD)

\begin{tabular}{lcccc}
\hline & Group GO & Group GD & Group GM & Group GX \\
\hline Anesthetic length (min) & $103.33 \pm 33.2$ & $93.42 \pm 26.88$ & $80.53 \pm 20.06$ & $89.74 \pm 15.05$ \\
PACU length (min) & $161.89 \pm 5.87$ & $163.90 \pm 7.40$ & $162.35 \pm 6.23$ & $100.37 \pm 24.35$ \\
\hline
\end{tabular}

There were no statistical differences among groups

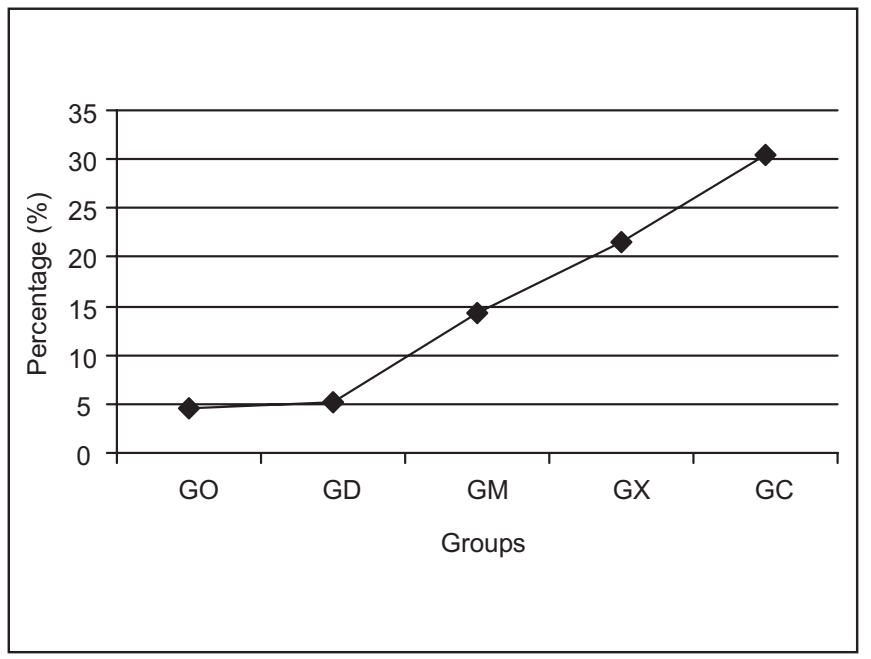

Figure 1 - Incidence of Nausea

\section{DISCUSSION}

The high incidence of vomiting after videolaparoscopic gynecological procedures was evident also in this study where control group patients had $26.09 \%$ vomiting. However, after postoperative vomiting treatment with metoclopramide, there has been no additional PACU or hospital stay, that is, there was no significant difference in PACU stay, corresponding to general anesthesia recovery stage II. Patients were referred to outpatient setting recovery (recovery-2) where they remained until hospital discharge (general anesthesia recovery stage III) ${ }^{11,12}$, when they no longer presented nausea and/or vomiting. Group's uniformity with regard to PACU discharge has also shown no influence of antiemetic drugs, especially droperidol in the dose used, in PACU stay. Hospital discharge within the estimated period of four hours after

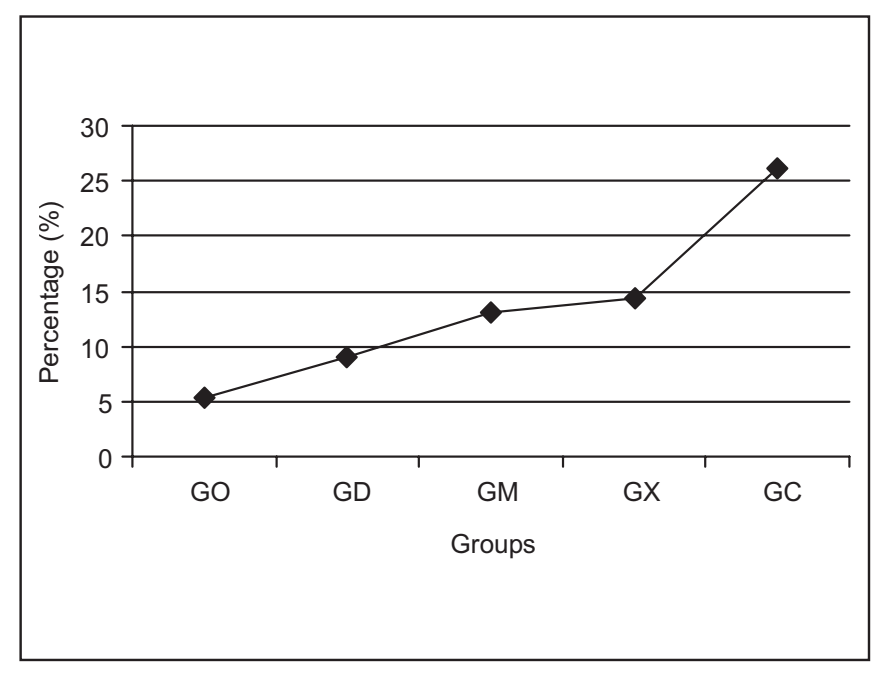

Figure 2 - Incidence of Vomiting

PACU discharge was achieved in all groups, also showing the efficacy of the anesthetic technique.

Ondansetron was the most effective drug in preventing postoperative nausea $(4.55 \%)$ and vomiting $(5.26 \%)$, which is in line with other authors ${ }^{8-10,13-15}$. From all drugs studied, droperidol was the closest to ondansetron in preventing nausea $(5.27 \%)$ and vomiting $(9.09 \%)$ after videolaparoscopic gynecological procedures, in the conditions of this study. Metoclopramide and dexametasone were, in this order, the less effective drugs. Alone, they have not promoted satisfactory results since none of them was able to significantly decrease the incidence of vomiting as compared to the control group. It is known that nausea and vomiting have a multifactorial etiology ${ }^{2,7}$ related to four types of neurotransmitters modulating the triggering chemoreceptor zone located in the postreme area: dopamine, serotonin, histamine and acetylcholine ${ }^{9}$.

Revista Brasileira de Anestesiologia Vol. 56, № 1, Janeiro - Fevereiro, 2006 
Antiemetic drugs are classified according to their action on pharmacological receptors and, in general, single therapy may not be enough to control PONV. Some authors suggest the association of two or more antiemetic agents to achieve better results ${ }^{16-18}$. A study has shown that prophylactic intravenous granisetron ( $3 \mathrm{mg}$ ) associated to dexametasone (8 $\mathrm{mg}$ ), were more effective than granisetron alone to decrease PONV in patients submitted to Cesarean section under spinal anesthesia ${ }^{16}$. The same authors have concluded, in a different study, that prophylactic therapy with granisetron and dexametasone was more effective than each antiemetic drug alone to prevent post-pediatric procedures vomiting ${ }^{17}$. Other authors have shown that the association of intravenous droperidol (1.25 mg) and ondansetron (4 mg) was significantly better than droperidol alone to control PONV in women submitted to laparoscopic gynecological procedures ${ }^{18}$. Our study has not combined antiemetic drugs since our aim was to study them separately.

So, in the conditions of our study where single antiemetic drugs were used, ondansetron was the most effective agent to prevent nausea and vomiting, and droperidol was the closest drug to ondansetron, representing an acceptable option to prevent nausea and vomiting after outpatient videolaparoscopic gynecological procedures.

\section{REFERÊNCIAS - REFERENCES}

01. Kenny GN - Risk factors for postoperative nausea and vomiting. Anaesthesia, 1994;49:(Suppl):6-10.

02. Schmidt A, Bagatini A - Náusea e vômito pós-operatório: fisiopatologia, profilaxia e tratamento. Rev Bras Anestesiol, 1997; 47:326-334.

03. Heyland K, Dangel P, Gerber AC - Postoperative nausea and vomiting (PONV) in chuldren. Eur J Pediatr Surg, 1997;7: 230-233.

04. Yuen HK, Chiu JW - Multimodal antiemetic therapy and emetic risck profiling. Ann Acad Med Singapore , 2005;34:196-205.

05. Lerman $\mathrm{J}$ - Surgical and patient factor involved in postoperative nausea and vomiting. $\mathrm{Br} J$ Anaesth, 1992;69:(Suppl7): 24S-32S.

06. Abreu MP - Náuseas e Vômitos - Antieméticos, em: Cangiani LM - Anestesia Ambulatorial, São Paulo, Atheneu, 2001;23:339-357

07. Ganem EM, Fabris P, Moro MZ et al - Eficácia do ondansetron e da alizaprida na prevenção de náusea e vômito em laparoscopia ginecológica. Rev Bras Anestesiol, 2001;51:401-406.

08. Koivuranta M, Ala-Kokko TI, Jokela R et al - Comparison of ondansetron and tropisetron combined with droperidol for the prevention of emesis in women with a history of post-operative náusea and vomiting. Eur J Anaesthesiol, 1999;16:390-395.

09. Domino KB, Anderson EA, Polissar NL et al - Comparative efficacy and safety of ondansetron, droperidol, and metoclopramide for preventing postoperative nausea and vomiting: a meta-analysis. Anesth Analg, 1999;88:1370-1379.

10. Mckenzie R, Kovac A, O'Connor T et al - Comparison of ondansetron versus placebo to prevent postoperative nausea and vomiting in women undergoing ambulatory gynecologic surgery. Anesthesiology, 1993;78:21-28.
11. Porto AM - Recuperação da Anestesia, em: Cangiani LM Anestesia Ambulatorial, São Paulo, Atheneu, 2001:311-322.

12. Porto AM - Critérios de Alta, em: Cangiani LM - Anestesia Ambulatorial, São Paulo, Atheneu, 2001:323-336.

13. Malins AF, Field JM, Nesling PM et al - Nausea and vomiting after gynaecological laparoscopy: comparison of premedication with oral ondansetron, metoclopramide and placebo. $\mathrm{Br} \mathrm{J}$ Anaesth, 1994;72:231-233.

14. Suen TK, Gin TA, Chen PP et al - Ondansetron $4 \mathrm{mg}$ for the prevention of nausea and vomiting after minor laparoscopic gynaecological surgery. Anaesth Intensive Care, 1994;22:142-146.

15. Raphael JH, Norton AC - Antiemetic efficacy of prophylactic ondansetron in laparoscopic surgery: randomized, double-blind comparison with metoclopramide. $\mathrm{Br} J$ Anaesth, 1993;71:845-848.

16. Fujii $\mathrm{Y}$, Saitoh $\mathrm{Y}$, Tanaka $\mathrm{H}$ et al - Granisetron/dexametasone combination for reducing nausea and vomiting during and after spinal anesthesia for cesarean section. Anesth Analg, 1999;88:1346-1350.

17. Fujii, Saitoh, Tanaka $\mathrm{H}$ et al - Prophylactic therapy with combined granisetron and dexametasone for the prevention of post-operative vomiting in children. Eur J Anaesthesiol, 1999;16:376-379.

18. Mckenzie R, Uy NT, Riley TJ et al - Droperidol/ondansetron combinations controls nausea and vomiting after tubal banding. Anesth Analg, 1996;83:1218-1222.

\section{RESUMEN}

Abreu MP, Vieira JL, Silva IF, Miziara LEPG, Fofano R - Eficacia de Ondansetron, Metoclopramida, Droperidol y Dexametasona en la Prevención de Nausea y Vómito Luego de Laparoscopía Ginecológica en Régimen Ambulatorial. Estudio Comparativo

JUSTIFICATIVA Y OBJETIVOS: Aunque el ondansetron es considerado una de las drogas mas eficientes para control de nausea y vómito post-operatorio (NVPO), su alto costo torna inviable su uso en forma rutinaria. Este estudio tuvo como finalidad verificar entre droperidol, metoclopramida $y$ dexametasona cual se aproxima más a la eficacia del ondansetron en la prevención de NVPO en laparoscopías ginecológicas.

MÉTODO: Participaron del estudio 100 pacientes sometidas a laparoscopía ginecológica con anestesia general intravenosa e inhalatoria, divididas aleatoriamente en cinco grupos de acuerdo con la medicación antiemética recibida. El grupo GO $(n=20)$ recibió ondansetron $(4 \mathrm{mg})$; el grupo $\mathrm{GM}(n=20)$ : metoclopramida $(10 \mathrm{mg})$; el grupo $G D(n=20)$ : droperidol $(1,25$ $m g)$, el grupo $G X(n=20)$ : dexametasona $(8 \mathrm{mg})$ y el grupo $G C$ grupo control $(n=20)$ no recibió medicación antiemética. Fueron verificadas la incidencia de nausea y/o vómito en el post-operatorio, los parámetros hemodinámicos, el tiempo en la sala de recuperación post-anestésica (SRPA) y el tiempo de la anestesia.

RESULTADOS: No hubo diferencia estadística entre los grupos en los registros antropométricos, hemodinámicos, tiempo de recuperación y de anestesia. Hubo diferencia estadística entre los grupos en la incidencia de nausea $(G O<$ $G D<G X<G M<G C)$ y vómito ( $G O<G D<G X<G M<G C)$.

CONCLUSIONES: En este estudio, el ondansetron fue el agente más eficaz para la prevención de nausea y vómito. El droperidol fue la droga que más se aproximó de la eficacia del ondansetron para prevenir NVPO. 